response relations which cannot satisfactorily account for all experimental results are scarcely worth apply. ing to the human case. In the absence of a satisfactory theory, it seems pointless to expend the enormous experimental effort required to define the relation between daily dose and life-span for mean survival-times of 95 per cent and more of the control : it is only in this region that extrapolation to man is of any particular interest.

I would like to thank my colleagues for allowing me to make use of unpublished material.

1 Boche, R. D. (1946), eited by him in "Biological Effects of External Radiation" (edit. H. A. Blair), NNES V1-2 (McGraw-Hill, New York, 1954)

2 Evans, T. C., Nucleonics, 4, 2 (1949).

Henshaw, P. S., Riley, E. F., and Stapleton, G. E., Radiology, 49, 349 (1947).

Lorenz, E., Jacobson, L. O., Heston, W. E., Shimkin, M., Eschenbrenner, A. B., Deringer, Margaret K., Doniger, Jane, and Schweisthal, R., in "Biological Effects of External $X$ - and Gamma-Radiation" (edit. R. E. Zirkle), N NES IV-2213 (MeGrawHill, New York, 1954).
'Sacher, G., J. Nat. Cancer Inst, 15, 1125 (1955). - Neary, G. J., Munson, R. J., and Mole, R. H., "Chronic Irradiation
of Mice by Fast Neutrons" (Pergamon Press, London, 1957), In the press.

? Irenshaw, P. S., J. Nat. Cancer Inst., 4, 513 (1944).

"Lorenz, E., in "Biological Effects of External X- and GammaRadiation" (edit. R. E. Zirkle), NNES IV-22B (McGraw-Hill, New York, 1954).

Blair, H. S., "Peaceful Uses of Atomic Energy", 11, 118 (United Nations, New York, 1956).

${ }^{16}$ Sacher, G., Radiology, 67, 250 (1956).

"Mole, R. H., J. Nat. Cancer Inst., 15, 907 (1955).

12 Sampford, M. R., Biometrics, 8, 13 and 307 (1952).

${ }^{13}$ Neary, G. J., Munson, R. J., Hulse, E. V., and Mole, R. H. (unpublished observations).

"Mole, R. H., in "Progress in Radiobiology", edit. J. S. Mitchell, B. E. Holmes and C. L. Smith (Oliver and Boyd, Edinburgh, 1956).

${ }^{15}$ Mole, R. H., Brit. J. Radiology, 30, 40 (1957).

16 Thompson, J. F., Tourtellotte, W. W., Carttar, M. S., Cox, jun., R. S., and Wilson, J. E., Amer. J. Roentgenology, 89, 830 (1953).

${ }^{17}$ Mole, R. H., Proc. VI Int. Hæmatology Congress, Boston (in the press).

${ }^{18}$ Mole, R. H., Brit. J. Radiology, 26, 234 (1953).

${ }^{10}$ Kaplan, H. S., Carnes, W. H., Brown, M. B., and Hirsch, B. B. Cancer Research, 16, 422 (1956).

\title{
LOW-TEMPERATURE PHYSICS IN THE U.S.S.R.
}

$\mathrm{T}$ THE fourth Russian conference on low-tem. perature physics was held during July 2-8 at the Institute for Physical Problems of the Soviet Academy of Sciences in Moscow. Six physicists from Great Britain, Drs. R. G. Chambers, H. A. Hall and A. B. Pippard from Cambridge, and Drs. R. Berman, A. H. Cooke and the author from Oxford, were invited to this meeting as guests of the Academy and were the only foreign visitors.

Since this was the first meeting with Russian lowtemperature workers, a brief enumeration of the Soviet cryogenic research centres may be of interest. There are two laboratories in Moscow, since, besides the Institute of the Academy, there is also a cryogenic research team under A. I. Shalnikov working at the University. Kapitza is the director of the Institute for Physical Problems, but in the past two years he has devoted himself to other than lowtemperature research. Peshkov is the deputy director and Molkov is in charge of the section dealing with cryogenic engineering. Another section, in a separate building, carries out research into the electronic behaviour of metals and is in the charge of Alekseevsky. Also at the Institute there is a theoretical physics section of which Landau is the head and in which E. M. Lifshitz and Khalatnikov are senior members. Kharkov has an old-established cryogenic laboratory, with Lazarev as director and I. M. Lifshitz and Kaganov as theoreticians. Of more recent date is the low-temperature laboratory at Tiflis, which is under the direction of Andronikashvili. There is another new cryogenic laboratory in Sverdlovsk.

At the conference, papers and discussions, except those by the visitors, were given in Russian; but the visitors were supplied with headphones and a simultaneous translation was provided for them. For this translation Kapitza had obtained the services of Mr. Boris Belitzky, of Moscow Radio, who is a physico-chemist by training. Mr. Belitzky's truly amazing gift for rendering not only the lectures but also the-often heated-arguments accurately into faultless English made all the difference to the success of the meetings so far as we were concerned. $\mathrm{He}$ clearly lived the part of the speakers, and the visitors came to forget that the voice they heard was not that of the lecturer. Judging from adverse reports on similar meetings, the value of a really good interpreter cannot be assessed too highly at a conference of this type. We were able to follow the proceedings so completely that a whole week of lectures in Russian never became tedious.

The following brief summary of the papers presented at the conference cannot give any details of the experimental results or the theoretical investigations which were reported. Its object is to give an idea of the type of research in progress in the U.S.S.R. and to provide a list of the work done by the various authors so that interested readers can apply to them for reprints or further information*.

After an opening address by Kapitza, the conference began with a series of papers on liquid helium which occupied two sessions. Much of the work was concerned with the light isotope and with isotope mixtures. The viscosity of pure liquid helium-3 has been measured between $0 \cdot 35^{\circ}$ and $3 \cdot 2^{\circ} \mathrm{K}$. by Miss K. N. Zinovieva, who works in Peshkov's laboratory. She reported a sharp rise of the viscosity with falling temperature below $1^{\circ} \mathrm{K}$. which, however, at the lowest measured temperature has not yet reached the theoretically predicted value of $T^{-2}$. Above $1^{\circ} \mathrm{K}$. a slight drop in viscosity with rising tem. perature is in qualitative agreement with the theory of Abrikosov and Khalatnikov. It is interesting to note that she also found a slight drop in the viscosity of helium-4 above $2 \cdot 6^{\circ} \mathrm{K}$. instead of the slight rise with temperature reported by Bowers and the present author seven years ago. She ascribed (correctly) this discrepancy to the omission of a correction in our work. Miss T. P. Ptukha, working in the same laboratory, presented measurements of the density of mixtures of liquid helium-3 and helium-4 and deduced lambda temperatures for four concentrations from the break in the density curves. The Kharkov workers, B. N. Eselson, A. D. Shvets and R. A. * The following addresses may be found useful: Institute for
Physical Problems of the Academy of Sciences, Kaloujskoe Shosse 32, Moscow; Physical Institute of the Ukrainian Academy of Sciences, Academy of Sciences, Allaverdovskaya Pl. 7, Tbilissi. 
Bablidze, have determined the lambda points of mixtures of helium-3 and helium-4 by the onset of film flow. An interesting theoretical investigation into the phenomena to be expected when first or second sound passes through a phase boundary between solutions of helium-3 and helium-4 has been made by R. G. Arkhipov and I. M. Khalatnikov. They gave the equations for reflexion, refraction and conversion from one type of sound into the other for the cases when both or only one of the phases are superfluid. In another theoretical paper, by L. P. Gorkov and L. P. Pitaevsky, the scattering of light in helium-3-helium-4 solutions was calculated, and it was shown that whereas pure helium-4 should exhibit only four lines, the spectrum of the solutions should contain five. A theory of the momentum distribution of interacting particles in Fermi systems was proposed by A. B. Migdal.

Except for some theoretical considerations of the helium film by R. G. Arkhipov and a report on normal viscosity and the onset of friction given by the present author, the rest of the work on helium was concerned with rotation experiments. Both H. A. Hall as well as D. S. Zakadze and E. L. Andronikashvili described oscillation experiments in rotating liquid helium. An investigation into the existence of discontinuity surfaces in moving liquid helium, postulated by Ginsburg, has been made by G. A. Gamzemlidze, who concludes that the surface energy of such discontinuities cannot exceed $10^{-9}$ erg $/ \mathrm{cm} .{ }^{2}$. A theoretical attempt to estimate the relaxation time of vortices in rotating helium has been made by Y. G. Mamaladze who, like the previous author, works in Tiflis. E. M. Lifshitz and L. P. Pitaevsky discussed the scatter of rotons on the vortex lines in rotating helium and have postulated that a strong interaction must exist between these two if the theory is to explain the experimental observations.

A good deal of the work on superconductivity dealt with the boundary conditions between the two states, using the Landau-Ginsburg theory as the guiding principle. The corrections due to relativistic magnetic interactions were taken into account by V. L. Ginsburg and L. V. Keldysh. The first author also presented a paper evaluating the spread of mag. netic field around the equilibrium value in which supercooling and overheating might be expected. Closely connected. with these considerations is an interesting theory of A. A. Abrikosov on the behaviour of superconductive alloys. The well-known fact of the gradual magnetic penetration over an interval of field values is considered as a basic property of the alloy rather than due to macroscopic inhomogeneities. Measurements of the surface tension between the superconductive and the normal state in very pure tin single crystals were reported by $\mathrm{Y}$. V. Sharvin, who found that the observed values are of the same order of magnitude but somewhat smaller than the values predicted by the Landau-Ginsburg theory. The conditions for the possible occurrence of superconductivity in bulk samples of ferromagnetic materials were discussed by G. F. Zharkov. He suggests the use of flat ellipsoids of rotation with a large demagnetization factor as most suitable for the detection of superconductivity.

The method of thermal waves for determinations of heat conductivities and specific heats has been employed by N. V. Zavaritsky, who investigated tin, aluminium and zine down to $0 \cdot 15^{\circ} \mathrm{K}$. Dependence of the thermal conduction on the shape and surface conditions of the specimens was observed as well as an exponential rise with temperature of the specific heat in all three metals. A survey of heat conductivity work on superconductors, carried out in Oxford, was given by the present author. B. G. Lazarev and A. I. Sudovtsov reported measurements of the volume change occurring in tin and lead when superconductivity is established in a magnetic field, and of the discontinuity of the expansion coefficient at this transition. They found good thermodynamical agreement with the data for the effect of pressure on the transition of these metals. The same authors, together with A. P. Smimov, have observed superconductivity in thin beryllium films which had been deposited on a helium-cooled surface, the transition occurring at about $8^{\circ} \mathrm{K}$. The depth of penetration in a very pure cadmium sample has been determined by $\mathrm{N}$. S. Khaikin, using a high-frequency method. The measurements were carried down to about $0 \cdot 2^{\circ} \mathrm{K}$., where a value of $1.5 \times 10^{-5} \mathrm{~cm}$. (much smaller than the anomalously high value given by Hein and Steele) was found. Similar measurements on films of tin and mercury have been made by Miss L. A. Prozorova, who concludes that the dependence upon temperature of the depth of penetration is in good agreement with the Landau-Ginsburg theory. Further theoretical considerations based on these results were reported by B. M. Bolotovsky.

A general survey of the possibilities of Fermi surface investigations in metals was given by $I$. M. Lifshitz, and this was followed by a paper by A. B. Pippard on the Fermi surface in copper. Two separate sets of measurements on the influence of pressure on the de Haas - van Alphen effect were reported. One, by N. B. Brandt and V. A. Ventzel, dealt with a detailed investigation on bismuth; while in the work of B. I. Verkin, I. M. Dmitrenko and B. G. Lazarev, zinc and tin were studied as well. The amplitude of oscillations of susceptibility is decreased in all cases by the application of pressure. Another approach to the determination of the Fermi surface, through cyclotron resonance in metals, was discussed theoretically by M. Y. Azbel and E. A. Kaner, who have amplified their former work by calculating the surface impedance as a function of field and temperature over a wide range of fields. Experimental data of cyclotron resonance in tin were presented by P. A. Bezugly and A. A. Galkin, and a paper on cyclotron resonance in bismuth was given by R. G. Chambers. The theory of the anomalous skin effect in metals in the infra-red was discussed by R. N. Gurzhi.

The resistance minimum in gold has been investigated in some detail by N. E. Alekseevsky and Y. P. Gaidukov who, from magneto-resistance observations and measurements of the Hall effect and of the magnetic properties, have formed the opinion that at about 8 k.oersteds a change in the metal takes place which, however, requires further elucidation. The galvano-magnetic properties of bismuth, tin and aluminium have been studied by N. E. Alekseevsky, N. B. Brandt and T. I. Kostina. P. G. Strelkov and Miss I. N. Kalinkina have measured the specific heat of bismuth from $0.3^{\circ}$ to $4.4^{\circ} \mathrm{K}$. and found that it is composed of a cubic term and a very small linear term. The effect of isotopes on the heat conduction of dielectric crystals was discussed by R. Berman. O. V. Klyavin and A. V. Stepanov gave data for the mechanical properties of a number of metals at liquid-helium temperatures. 
The last session of the conference dealt with magnetic phenomena. The energy spectrum of antiferromagnetics near the ground-state was discussed by M. I. Kaganov and V. M. Zukernik. A. H. Cooke reported on antiferromagnetism in some iridium compounds, and antiferromagnetism in anhydrous sulphates and carbonates of the transition metals was described by A. S. Borovik-Romanov, N. M. Kreines and Miss M. P. Orlova. The work of the latter authors has led I. Dzyaloshinsky to a theory of weak ferromagnetism in antiferromagnetic crystals which, based on Landau's ideas, explains the effect as due to relativistic spin-lattice and magnetic dipole interactions. The propagation of sound in ferromagnetics was considered by A. I. Akhiezer, B. G. Baryakhtar and S. B. Peletminsky, who postulate that it should be possible to excite magnetic waves by means of sound, and that under certain stated conditions this should be an easily noticed effect.

Two afternoons of the conference were devoted to visits to the cryogenic laboratories at the Institute for Physical Problems and at the University of Moscow. An interesting historical exhibit at the former was the first turbine used by Kapitza in the liquefaction of air. Among the various research installations on show, a helium-3 cryostat in Peshkov's laboratory was particularly impressive. In this ingenious device five litres (at N.T.P.) of pure helium-3, which forms the working substance, is circulated constantly. The gas condenses under pressure in helium-4 and is then expanded. Temperatures down to $0 \cdot 3^{\circ} \mathrm{K}$. can be obtained in this way and temperatures down to $0.5^{\circ} \mathrm{K}$. can be maintained constant for indefinite periods. Peshkov and Miss Zinovieva showed us a photograph, taken in this apparatus, in which the separation of the two liquid phases of helium-3-helium-4 mixtures was clearly visible. At the University of Moscow we saw besides the research laboratories the cryogenic teaching course, where undergraduates were busy with their ten experiments involving liquid helium. One of them was just carrying out an adiabatic demagnetization.

The members of the conference came to know each other well on a whole day's boat trip arranged by Kapitza. The hospitality of the Academy and the friendly welcome of our Russian colleagues made our stay in Moscow a very pleasant one. The visitors expressed the hope that they will be able to induce their hosts soon to come to Britain for a return visit. K. Mendelssohn

\section{OBITUARIES}

Prof. H. O. Wieland, For.Mem.R.S.

TO-DAY the manufacture of steroids for medical purposes is an industry. Hundreds of scientists in academic and industrial life are engaged in research on these substances. All this activity properly began in Germany, and its modern phase really dates from the work of A. Windaus on cholesterol and other sterols, which began in 1903, and that of Heinrich Wieland, whose paper (with F. J. Weil) on the preparation of the 'stem' bile acid, cholanic acid, $\mathrm{C}_{24} \mathrm{H}_{40} \mathrm{O}_{2}$, was published in 1912 (Hoppe-Seyl. Z., 80, 287). Until 1949, fundamental discoveries, mainly about the occurrence in Nature and the chemical structures of bile acids, toad poisons and some sterols and alkaloids were published continually from Wieland's laboratories. These contributions have been reviewed, for example, by L. F. Fieser and M. Fieser ("Natural Products related to Phenanthrene". Reinhold, New York).

The award of a Nobel Prize in Chemistry to Wieland in 1927 and to Windaus in 1928 anticipated our full appreciation of the importance of their work and of the use which would be made of it.

When O. Rosenheim and H. King in 1932 (Chem. and Indust., 51, 464) put forward their revolutionary chrysene structure for sterols and bile acids, Wieland and E. Dane (Hoppe-Seyl. Z., 210, $268 ;$ 1932) were quick to see its advantages and almost at once proposed the formula now accepted. Wieland and his colleagues went on to show how this formula explained observations which they and the school of Windaus had collected for nearly thirty years.

At that time, the structures of the crystalline oestrogenic hormones which had been isolated independently by E. A. Doisy, C. D. Veler and S. Thayer (œstrone, 1929), G. F. Marrian (œstriol, 1930) and A. Butenandt (œstrone, 1929) from human pregnancy urine were still unknown; a leap in our under- standing followed the demonstrations that these hormones were related to cholesterol and the bile acids. The androgens, progesterone and the adrenal cortex hormones soon appeared in the same pattern. Antirachitic vitamins D were derived from ergosterol and, later, cholesterol. When Wieland and Dane (Hoppe-Seyl.Z., 219, 240; 1933) and J.W. Cook and his associates, at the same time, converted a bile acid (deoxycholic) into a powerfully carcinogenic hydrocarbon (methylcholanthrene), the foundations of much present-day interest in steroids had been laid.

Many new sterols and bile acids have since been discovered, and we now know that the heart poisons of toads and in certain plants, some plant sapogenins and alkaloids, as well as triterpenes of diverse origin, can be regarded as steroids. We have become adept at converting common sterols and sapogenins like cholesterol, diosgenin and hecogenin into known hormones and $D$ vitamins, as well as into other substances with many kinds of physiological activity. The principal steroids have been totally synthesized. Many chemical reactions of these substances can now be interpreted in terms of electronic theory. We have begun to understand how sterols are synthesized in vivo from simple substances like acetate and how sterols can give rise to bile acids and hormones. Studies of the metabolism of such hormones in the living body are far advanced. We grope towards comprehension of the significance of sterols themselves in living cells and of the means by which steroid hormones may affect cellular chemical reactions.

An immense amount of work remains to be done. No doubt fundamental changes in our appreciation of vital processes must follow or precede more complete understanding of the natural significance of steroids. 